\title{
Sequential Universal Modeling for Non-Binary Sequences with Constrained Distributions
}

$\begin{array}{lll}\begin{array}{l}\text { Michael Drmota } \\ \text { Institut für Geometrie }\end{array} & \text { Gil I. Shamir } & \text { Wojciech Szpankowski }{ }^{1} \\ \text { TU Wien } & & \text { Dept. of Computer Science } \\ \text { A-1040 Wien } & \text { Pittsburgh, PA } & \text { Purdue University } \\ \text { Austria } & \text { W.S.A. Lafayette, IN } 47907 \\ \text { drmota@tuwien.ac.at } & \text { gshamir@ieee.org } & \text { U.S.A. } \\ & \text { szpan@purdue.edu }\end{array}$

Michael Drmota

ghamir@ieee.org

\author{
Wojciech Szpankowski ${ }^{1}$ \\ Purdue University \\ W. Lafayette, IN 47907 \\ szpan@purdue.edu
}

\begin{abstract}
Sequential probability assignment and universal compression go hand in hand. We propose sequential probability assignment for non-binary (and large alphabet) sequences with distributions whose parameters are known to be bounded within a limited interval. Sequential probability assignment algorithms are essential in many applications that require fast and accurate estimation of the maximizing sequence probability. These applications include learning, regression, channel estimation and decoding, prediction, and universal compression. On the other hand, constrained distributions introduce interesting theoretical twists that must be overcome in order to present efficient sequential algorithms. Here, we focus on universal compression for memoryless sources, and present a precise analysis for the maximal minimax and the (asymptotic) average minimax redundancy for constrained distributions. We show that our sequential algorithm based on modified Krichevsky-Trofimov (KT) estimator is asymptotically optimal up to $O(1)$ for both maximal and average redundancies. In addition, we provide precise asymptotics of the minimax redundancy for monotone distributions which is a special case of the constrained distribution. This paper follows and addresses the challenge presented in [17] that suggested 'results for the binary case lay the foundation to studying larger alphabets".
\end{abstract}

Key Words: information theory, minimax redundancy, constrained distribution, analytic combinatorics

\section{Introduction}

Universal coding and universal modeling (probability assignments) are two driving forces of information theory, model selection, and statistical inference. In universal coding one is to construct a code for data sequences generated by an unknown source from a known family such that, as the length of the sequence increases, the average code length approaches the entropy of whatever processes in the family has generated the data. In seminal works of Davisson [4], Rissanen [12], Krichevsky and Trofimov [9], and Shtarkov [13] it was shown how to construct such codes for finite alphabet sources. Universal codes are often characterized by the average minimax redundancy which is the excess over the entropy of the best code from a class of decodable codes for the worst process in the family.

As pointed out by Rissanen [12], over years universal coding evolved into universal modeling where the purpose is no longer restricted to just coding but rather to learn optimal models

\footnotetext{
${ }^{1}$ M. Drmota was supported in part by the the grant FWF Grant SFB F50-02. W. Szpankowski was supported in part by NSF Center on Science of Information Grants CCF-0939370 and NSF Grants CCF-1524312, CCF2006440, CCF-2007238, and Google Research Award.
} 
[12]. The central question of interest in universal modeling seems to be in universal codes achievable for individual sequences. The burning question is how to measure its performance. The worst case minimax redundancy became handy since it measures the worst case excess of the best code maximized over the processes in the family. Unfortunately, low-complexity universal codes that are optimal for the worst case minimax are not easily implementable (it would require to implement the maximum likelihood distribution). Therefore, we design a sequential algorithm based on the KT-estimator that is asymptotically optimal on average (i.e., for the average minimax redundancy), and show that both redundancies differ by a small constant.

In this paper we focus on universal compression and probability assignment/learning for a class of memoryless sources with constrained distributions. Let us start with some definitions and notation. We define a code $C_{n}: \mathcal{A}^{n} \rightarrow\{0,1\}^{*}$ as a mapping from the set $\mathcal{A}^{n}$ of all sequences $x^{n}=\left(x_{1}, \ldots, x_{n}\right)$ of length $n$ over the finite alphabet $\mathcal{A}=\{1, \ldots, m\}$ of size $m$ to the set $\{0,1\}^{*}$ of all binary sequences. Given a probabilistic source model, we let $P\left(x^{n}\right)$ be the probability of the message $x^{n}$; given a code $C_{n}$, we let $L\left(C_{n}, x^{n}\right)$ be the code length for $x^{n}$. However, in practice the probability distribution (i.e., source) $P$ is unknown, and one looks for universal codes for which the redundancy is $o(n)$ for all $P \in \mathcal{S}$ where $\mathcal{S}$ is a class of source models (distributions). It is convenient to ignore the integer nature of the code length and replace it by its best distributional guess, say $Q\left(x^{n}\right)$. In other words, we just write $L\left(C_{n}, x^{n}\right)=-\log Q\left(x^{n}\right)$ and use it throughout the paper. The question is how well $Q$ approximates $P$ within the class $\mathcal{S}$. Minimax redundancy enters. Usually, we consider two types of minimax redundancy, namely average and maximal or worst case defined, respectively, as

$$
\begin{aligned}
& \bar{R}_{n}(\mathcal{S})=\min _{Q} \sup _{P \in \mathcal{S}} \mathbf{E}\left[\log P\left(X^{n}\right) / Q\left(X^{n}\right)\right], \\
& R_{n}^{*}(\mathcal{S})=\min _{Q} \sup _{P \in \mathcal{S}} \mathbf{m a x}_{x^{n}}\left[\log P\left(x^{n}\right) / Q\left(x^{n}\right)\right] .
\end{aligned}
$$

In this paper we analyze precisely both redundancies for memoryless sources over $m$-ary alphabet $\mathcal{A}=\{1, \ldots, m\}$ with restricted symbol probability $\theta_{i}$, that is, we assume that $\boldsymbol{\theta} \in \mathcal{S}$, where $\mathcal{S}$ is a proper subset of

$$
\Theta=\left\{\boldsymbol{\theta}: \theta_{i} \geq 0(1 \leq i \leq m), \theta_{1}+\cdots+\theta_{m}=1\right\} .
$$

We will assume that $\mathcal{S}$ is a convex polytope. As a special case we have the interval restriction $0 \leq a_{i} \leq \theta_{i} \leq b_{i} \leq 1$ for $i=1, \ldots m-1$, where $\sum_{i=1}^{m-1} b_{i} \leq 1$ (this ensures that $\theta_{m}=1-\sum_{i=1}^{m-1}$ is always well defined). Also, a class of monotone distributions [16] defined as

$$
\mathcal{M}=\left\{\boldsymbol{\theta}: 0 \leq \theta_{1} \leq \theta_{2} \leq \cdots \leq \theta_{m}, \theta_{1}+\cdots+\theta_{m}=1\right\}
$$

is a special case of the constrained distributions.

Here, we present a sequential algorithm that estimates asymptotically the optimal probability $P\left(x^{n}\right)$ for all $x^{n}$. It turns out that restricting the set of parameters is important from a practical point of view and at the same time introduces new interesting theoretical twists that we explore in this paper. We first prove in Theorem 1 that (for fixed $m$ that can still be large)

$$
\bar{R}_{n}(\mathcal{S})=R_{n}^{*}(\mathcal{S})+O(1)=\frac{m-1}{2} \log (n)+O(1)
$$

where the constant implied by the $O$-term depends on $m$ and on the set $\mathcal{S}$. Second we provide in Theorem 2 precise asymptotics for $\bar{R}_{n}(\Theta)$ and $R_{n}^{*}(\Theta)$ if $m=o(n)$. While the leading 
terms of these redundancies are known $[10,14,15,20]$, we derive here precise asymptotics up to $O\left(m^{3 / 2} / \sqrt{n}\right)$ term in a uniform manner that can be used to extend our analysis to the constrained case in this regime. This allows us in Theorem 3 to provide the best asymptotic expansions for the minimax redundancy of monotone distributions. Finally, we present in Corollary 1 a sequential add-1/2 KT-like estimator to compute $P\left(x_{n+1} \mid x^{n}\right)$ for the constrained distributions that is asymptotically optimal up to a constant for both the maximal and average redundancy. This final result has been wanting since [17] which suggested that "results for the binary case lay the foundation to studying larger alphabets".

This paper is organized as follows. In the next section we present our main results, including the sequential algorithm that directly generalizes the add-1/2-KT estimator. The proofs are discussed in the last section and an appendix.

\section{Main Results}

In this section we present our main results including asymptotically optimal probability estimation for the class $\mathcal{S} \subset \Theta$ of memoryless sources with constrained distributions.

We start with the worst case redundancy defined in (2). We recall that the distribution $P\left(x^{n}\right)$ is of the form

$$
P\left(x^{n}\right)=\prod_{i=1}^{m} \theta_{i}^{k_{i}}, \quad \theta_{i} \geq 0, \sum_{i=1}^{m} \theta_{i}=1,
$$

where $k_{i}$ is the number of symbol $i \in \mathcal{A}$ in the sequence $x^{n}$. The probabilities $\theta_{i}$ are unknown to us except that we restrict them to the subset $\mathcal{S} \subseteq \Theta$. Following Shtarkov [13] and [5] we can re-write the worst case redundancy for $\mathcal{S}$, by noting that max and sup commute, as

$$
\begin{aligned}
R_{n}^{*}(\mathcal{S}) & =\min _{Q} \sup _{P \in \mathcal{S}} \max _{x^{n}}\left(-\log Q\left(x^{n}\right)+\log P\left(x^{n}\right)\right) \\
& =\min _{Q} \max _{x^{n}}\left[-\log Q\left(x^{n}\right)+\sup _{P \in \mathcal{S}} \log P\left(x^{n}\right)\right] \\
& =\min _{Q} \max _{x_{n}}\left[\log Q^{-1}\left(x^{n}\right)+\log P^{*}\left(x^{n}\right)+\log \sum_{z^{n}} \sup _{P \in \mathcal{S}} P\left(z^{n}\right)\right] \\
& =\log \sum_{x^{n}} \sup _{P \in \mathcal{S}} P\left(x^{n}\right)
\end{aligned}
$$

where $P^{*}\left(x^{n}\right)$ is

$$
P^{*}\left(x^{n}\right):=\frac{\sup _{P \in \mathcal{S}} P\left(x^{n}\right)}{\sum_{z_{1}^{n}} \sup _{P \in \mathcal{S}} P\left(z_{1}^{n}\right)}
$$

is the maximum-likelihood distribution and we set $Q\left(x^{n}\right)=P^{*}\left(x^{n}\right)$ for attaining the minimum. In this context the distribution $P^{*}$ is also called Shtarkov distribution and the sum

$$
D_{n}=\sum_{x^{n}} \sup _{P \in \mathcal{S}} P\left(x^{n}\right)
$$

is called Shtarkov sum. Note that $R_{n}^{*}(\mathcal{S})=\log D_{n}$.

If we define the worst case redundancy with the help of code lengths $L\left(C_{n}, x^{n}\right)$ instead of $-\log Q\left(x^{n}\right)$ - that we denote by $\widetilde{R}_{n}^{*}$ - then we would get a similar expression of the form $\widetilde{R}_{n}^{*}(\mathcal{S})=\log D_{n}+\widetilde{R}_{n}^{*}\left(P^{*}\right)$. Using Shannon's code Shtarkov immediately noticed that $0<$ 
$\widetilde{R}_{n}^{*}\left(P^{*}\right)<1$, and in [5] it was actually proved that asymptotically for the unconstrained case

$$
\widetilde{R}_{n}^{*}\left(P^{*}\right)=\frac{\log \left(\frac{1}{m-1} \log m\right)}{\log m}+o(1) .
$$

From now on we shall ignore this correction term and analyze $R_{n}^{*}(\mathcal{S})=\log D_{n}$.

To estimate the sum $D_{n}=\sum_{x^{n}} \sup _{P} P\left(x^{n}\right)$ we need first to find $\sup \prod_{i=1}^{m} \theta_{i}^{k_{i}}$ when $\boldsymbol{\theta} \in \mathcal{S}$. For the unrestricted case $(\mathcal{S}=\Theta)$ we know that the optimal $\theta_{i}=k_{i} / n$. The situation is more complicated in the constrained case. For example, if we assume an interval restriction $a_{i} \leq \theta_{i} \leq b_{i}, i=1, \ldots, m-1$ with $\theta_{m}=1-\theta_{1}-\cdots-\theta_{m-1}$, then for $k_{i}<n a_{i}$ or $k_{i}>n b_{i}$ the optimal $\theta_{i}$ may be $a_{i}$ or $b_{i}$, respectively. Fortunately, we are able to prove that the main contribution to $D_{n}$ comes from those $\mathbf{k}=\left(k_{1}, \ldots, k_{m}\right)$ for which $\mathbf{k} / n \in \mathcal{S}$ (see (23) and Appendix). So we are led to analyze the following sum

$$
D_{n}^{(\mathcal{S})}=\sum_{\mathbf{k} \in n \mathcal{S}}\left(\begin{array}{c}
n \\
k_{1}, \cdots k_{m}
\end{array}\right) \prod_{i=1}^{m}\left(\frac{k_{i}}{n}\right)^{k_{i}}
$$

which is of order $n^{\frac{m-1}{2}}$. The contribution of the remaining terms is typically of order $O\left(n^{\frac{m-2}{2}}\right)$.

It is our goal to present a sequential low-complexity algorithm for the probability assignment, that is, an iterative procedure to compute $P\left(x_{n+1} \mid x^{n}\right)$. Unfortunately, the maximumlikelihood distribution (3) is not well suited for it. To find one, we switch to the average minimax redundancy (1) and we re-cast in the Bayesian framework.

Before we discuss the average minimax redundancy, we need to introduce one more notation element. Let us define the Dirichlet density as

$$
\operatorname{Dir}\left(\theta_{1}, \ldots, \theta_{m} ; \alpha_{1}, \ldots, \alpha_{m}\right)=\frac{1}{B\left(\alpha_{1}, \ldots, \alpha_{m}\right)} \prod_{i=1}^{m} \theta_{i}^{\alpha_{i}-1},
$$

where $\sum_{i=1}^{m} \theta_{i}=1$ and

$$
B\left(\alpha_{1}, \ldots, \alpha_{m}\right)=B_{m}\left(\alpha_{1}, \ldots, \alpha_{m}\right)=\frac{\Gamma\left(\alpha_{1}\right) \cdots \Gamma\left(\alpha_{m}\right)}{\Gamma\left(\alpha_{1}+\cdots+\alpha_{m}\right)}
$$

is the beta function. We shall write $\boldsymbol{\alpha}=\left(\alpha_{1}, \ldots \alpha_{m}\right)$ and $\boldsymbol{\theta}=\left(\theta_{1}, \ldots \theta_{m}\right)$ with $\sum_{i=1}^{m} \theta_{i}=1$. Finally, we set for $\mathcal{S} \subset \Theta$

$$
\operatorname{Dir}(\mathcal{S} ; \boldsymbol{\alpha})=\frac{1}{B(\boldsymbol{\alpha})} \int_{\mathcal{S}} \boldsymbol{\theta}^{\boldsymbol{\alpha}-1} d \boldsymbol{\theta}
$$

Let $\mathcal{S} \subseteq \Theta$. Then the average minimax problem is

$$
\bar{R}_{n}(\mathcal{S})=\inf _{Q} \sup _{\theta \in \mathcal{S}} D_{n}\left(P^{\theta} \| Q\right)
$$

where $D\left(P^{\theta} \| Q\right)$ is the Kullback-Leibler divergence. In the Bayesian framework, one assumes that the parameter $\theta$ is generated by the density $w(\theta)$ and the mixture $M_{n}^{w}\left(x^{n}\right)$ is

$$
M_{n}^{w}\left(x^{n}\right)=\int_{\mathcal{S}} P^{\theta}\left(x^{n}\right) w(d \theta)
$$

Observe now

$$
\begin{aligned}
\inf _{Q} \mathbf{E}_{w}\left[D_{n}\left(P^{\theta} \| Q\right)\right] & =\inf _{Q} \int_{\mathcal{S}} D_{n}\left(P^{\theta} \| Q\right) d w(\theta) \\
& =\int_{\mathcal{S}} D_{n}\left(P^{\theta} \| M_{n}^{w}\right) d w(\theta)
\end{aligned}
$$


where we use the fact that

$$
\min _{Q} \sum_{i} P_{i} \log 1 / Q_{i}=\sum_{i} P_{i} \log 1 / P_{i}
$$

As pointed out by Gallager [7], and Davisson [4] the minimax theorem of game theory entitles us to conclude that

$$
\bar{R}_{n}(\mathcal{S})=\inf _{Q} \sup _{\theta \in \mathcal{S}} D_{n}\left(P^{\theta} \| Q\right)=\sup _{w} \inf _{Q} \mathbf{E}_{w}\left[D_{n}\left(P^{\theta} \| Q\right)\right]
$$

leading to

$$
\bar{R}_{n}(\mathcal{S})=\int_{\mathcal{S}} D\left(P^{\theta} \| M_{n}^{w^{*}}\right) d w^{*}(\theta)
$$

where $w^{*}(\theta)$ is the maximizing prior distribution. Bernardo [1] proved that asymptotically the maximizing density is proportional to the square root of the determinant of the Fisher information $I(\boldsymbol{\theta})$, the so-called Jeffrey prior. This leads to the density

$$
\widetilde{w}^{*}(\boldsymbol{\theta})=\frac{1}{C(\mathcal{S}) \cdot B(\mathbf{1} / \mathbf{2})} \frac{1}{\sqrt{\theta_{1} \cdots \theta_{m}}},
$$

where $C(\mathcal{S})$ defined as

$$
C(\mathcal{S})=\operatorname{Dir}(\mathcal{S} ; \mathbf{1} / \mathbf{2})=\frac{1}{B(\mathbf{1} / \mathbf{2})} \int_{\mathcal{S}} \frac{d \boldsymbol{\theta}}{\sqrt{\theta_{1} \cdots \theta_{m}}}
$$

is the probability that the Dirichlet distribution with $\alpha_{i}=1 / 2$ falls into the subset $\mathcal{S}$. For example, Clarke and Barron [2] showed (under proper regularity conditions are satisfied, including the finiteness of the determinant of Fisher information and that $\mathcal{S}$ is a compact subset of the interior of $\Theta$ ) that

$$
\begin{aligned}
& \lim _{n \rightarrow \infty}\left(\int_{\mathcal{S}} D\left(P^{\theta} \| M_{n}^{w^{*}}\right) d w^{*}(\theta)-\frac{m-1}{2} \log \frac{n}{2 \pi e}\right) \\
& =\lim _{n \rightarrow \infty}\left(\int_{\mathcal{S}} D\left(P^{\theta} \| M_{n}^{\widetilde{w}^{*}}\right) d \widetilde{w}^{*}(\theta)-\frac{m-1}{2} \log \frac{n}{2 \pi e}\right)=\log \int_{\mathcal{S}} \sqrt{\operatorname{det} I(\boldsymbol{\theta})} d \boldsymbol{\theta} .
\end{aligned}
$$

Barron and Xie [22] extended this result to the unconstrained case $\mathcal{S}=\Theta$. We note that $C(\Theta)=1$ for the unconstrained case.

This leads us to the following notation of the asymptotic average minimax redundancy

$$
\bar{R}_{n}^{\text {asymp }}(\mathcal{S})=\int_{\mathcal{S}} D\left(P^{\boldsymbol{\theta}} \| M_{n}^{\widetilde{w}^{*}}\right) d \widetilde{w}^{*}(\boldsymbol{\theta}) .
$$

The mixture distribution $M_{n}^{\widetilde{w}^{*}}\left(x^{n}\right)$ can be calculated as follows

$$
\begin{aligned}
M_{n}^{\widetilde{w}^{*}}\left(x^{n}\right) & =\frac{1}{C(\mathcal{S}) \cdot B(\mathbf{1} / \mathbf{2})} \int_{\mathcal{S}} \prod_{i=1}^{m} \theta_{i}^{k_{i}-1 / 2} \\
& =\frac{1}{C(\mathcal{S}) \cdot B(\mathbf{1} / \mathbf{2})} B\left(k_{1}+1 / 2, \cdots, k_{m}+1 / 2\right) \\
& \cdot \frac{1}{B\left(k_{1}+1 / 2, \cdots, k_{m}+1 / 2\right)} \int_{\mathcal{S}} \prod_{i=1}^{m} \theta_{i}^{k_{i}-1 / 2} \\
& =\frac{1}{C(\mathcal{S}) \cdot B(\mathbf{1} / \mathbf{2})} B\left(k_{1}+1 / 2, \cdots, k_{m}+1 / 2\right) \cdot \operatorname{Dir}(\mathcal{S}: \mathbf{k}+\mathbf{1} / \mathbf{2}) .
\end{aligned}
$$


Observe again that for the unconstrained case $\operatorname{Dir}(\Theta ; \mathbf{k}+1 / 2)=1$. In summary

$$
\begin{aligned}
D_{n}\left(P^{\theta} \| M_{n}^{\widetilde{w}^{*}}\right) & =\log (C(\mathcal{S}) B(\mathbf{1} / \mathbf{2}))+ \\
& +\sum_{\mathbf{k}}\left(\begin{array}{l}
n \\
\mathbf{k}
\end{array}\right) \prod_{i=1}^{m} \theta_{i}^{k_{i}} \log \frac{\prod_{i=1}^{m} \theta_{i}^{k_{i}}}{B(\mathbf{k}+\mathbf{1} / \mathbf{2}) \operatorname{Dir}(\mathcal{S} ; \mathbf{k}+\mathbf{1} / \mathbf{2})}
\end{aligned}
$$

We are now ready to formulate our first main result that reads as follows. We prove them in the next section and delay some technical derivations to Appendix.

Theorem 1. Consider a memoryless constrained source $\mathcal{S} \subset \Theta$ with fixed but arbitrarily large $m \geq 2$ where $\mathcal{S}$ is a convex polytope. Then the worst case minimax redundancy for $\mathcal{S}$ is

$$
\begin{aligned}
R_{n}^{*}(\mathcal{S}) & =\frac{m-1}{2} \log (n / 2)-\log \Gamma(m / 2)+\log C(\mathcal{S}) \\
& +\frac{1}{2} \log \pi+O(1 / \sqrt{n})
\end{aligned}
$$

and the corresponding asymptotic average minimax redundancy is

$$
\begin{aligned}
\bar{R}_{n}^{\text {asymp }}(\mathcal{S}) & =\frac{m-1}{2} \log (n / 2 e)-\log \Gamma(m / 2)+\log C(\mathcal{S}) \\
& +\frac{1}{2} \log \pi+O(1 / \sqrt{n})
\end{aligned}
$$

where, we recall,

$$
C(\mathcal{S})=\operatorname{Dir}(\mathcal{S} ; \mathbf{1} / \mathbf{2})=\frac{1}{B(\mathbf{1} / \mathbf{2})} \int_{\mathcal{S}} \frac{d \boldsymbol{\theta}}{\sqrt{\theta_{1} \cdots \theta_{m}}}
$$

as defined above in (6) with $C(\Theta)=1$.

We observe that $R_{n}^{*}(\mathcal{S})$ and $\bar{R}_{n}^{\text {asymp }}(\mathcal{S})$ differ approximately by $\frac{m-1}{2}$. This fact should be compared with a general results of $[5$, Theorem 6$]$ where it was proved that for a large class of sources

$$
\left|\bar{R}_{n}(\mathcal{S})-\bar{R}_{n}^{*}(\mathcal{S})\right| \leq c_{n}(\mathcal{S})
$$

where

$$
c_{n}(\mathcal{S})=\sup _{P \in \mathcal{S}} \sum_{x^{n}} P\left(x_{1}^{n}\right) \lg \frac{\sup _{P \in \mathcal{S}} P\left(x_{1}^{n}\right)}{P\left(x_{1}^{n}\right)} .
$$

Actually, for binary memoryless sources $c_{n}(\mathcal{S}) \leq 1$ and $c_{n}(\mathcal{S}) \leq m-1$ for $m$-ary memoryless sources ([5, Lemma 8] extends directly to the $m$-ary case).

In Theorem 1 we assumed that $m$ is fixed to avoid complications with constrains $\mathcal{S}_{m}$ that may depend on $m$. In this paper we present our results for large $m=o(n)$. While the leading terms, especially for the maximal minimax redundancy, were known before (see $[11,10,14,15,20,21,22])$, our results are derived in a novel way that allows us to apply the methodology to obtain in Theorem 3 best redundancy results for monotone distributions over large alphabet (see [16]).

Theorem 2. Consider a memoryless unconstrained source $\Theta$ with $m=o(n)$. Then the unconstrained maximal redundancy is

$$
R_{n}^{*}(\Theta)=\frac{m-1}{2} \log \left(\frac{e n}{m}\right)+\frac{1}{2}(1-\log 2)+O(1 / m)+O\left(m^{3 / 2} / \sqrt{n}\right) .
$$

and the unconstrained asymptotic average redundancy becomes

$$
\bar{R}_{n}^{\text {asymp }}(\Theta)=\frac{m-1}{2} \log \left(\frac{n}{m}\right)+\frac{1}{2}(1-\log 2)+O(1 / m)+O\left(m^{3 / 2} / \sqrt{n}\right) .
$$


Remark 1. In order to compare Theorems 1 and 2 we need to set $m$ fixed in Theorem 2 which means to keep $\Gamma(m / 2)$ as in $(22)$ and set $C(\Theta)=1$.

Monotone Distributions. As an application of Theorems 1-2 we provide precise asymptotics for monotone distributions which can be viewed as a special case of the constrained distribution. Indeed, recall

$$
\mathcal{M}=\left\{\boldsymbol{\theta} \in \Theta: \theta_{1} \leq \theta_{2} \leq \cdots \leq \theta_{m}\right\} .
$$

Then the unconstrained set $\Theta$ can be divided into $m$ ! subsets

$$
\mathcal{M}_{\pi}=\left\{\boldsymbol{\theta} \in \Theta: \theta_{\pi(1)} \leq \theta_{\pi(2)} \leq \ldots \leq \theta_{\pi(m)}\right\}
$$

for any permutation $\pi$ of $\{1,2, \ldots m\}$. Furthermore, for all symmetric functionals $f$ it is easy to see that

$$
\int_{\mathcal{M}} f\left(\theta_{1}, \ldots, \theta_{m}\right) d \theta_{1} \ldots d \theta_{m}=\frac{1}{m !} \int_{\Theta} f\left(\theta_{1}, \ldots, \theta_{m}\right) d \theta_{1} \ldots d \theta_{m}
$$

More precisely, let $D_{n}(\Theta)$ and $D_{n}(\mathcal{M})$ denote the Shtarkov sums for the the unconstrained distribution and monotone distribution, respectively. Then in Section 3.3 we basically show that (see (28))

$$
D_{n}(\mathcal{M})=\frac{1}{m !} D_{n}(\Theta)+O\left((2 \pi)^{-\frac{m-1}{2}} m^{3 / 2} n^{\frac{m-2}{2}} B_{m}(\mathbf{1} / \mathbf{2}) \exp \left(O\left(\frac{m^{3 / 2}}{\sqrt{n}}\right)\right)\right) .
$$

This leads to our next main result regarding the redundancy for monotone distribution and large alphabet. To the best of our knowledge this is the most precise asymptotic expansion (up to $O(1)$ term) for $m=o(\log n / \log \log n)$ (cf. [16]).

Theorem 3. Consider a class of monotone distributions $\mathcal{M}$. Then $R_{n}^{*}(\mathcal{M})=R_{n}^{*}(\Theta)-\log m !+$ $O\left(m ! m^{3 / 2} / \sqrt{n}\right)$. In particular, for $m=o(n)$ we have

$$
\begin{aligned}
R_{n}^{*}(\mathcal{M}) & =\frac{m}{2} \log \left(\frac{n}{m^{3}}\right)+\frac{3}{2} m \log e+\frac{1}{2} \log \frac{2 \pi m}{e}+\frac{1}{2}(1-\log 2) \\
& +O(1 / m)+O\left(m ! m^{3 / 2} / \sqrt{n}\right)
\end{aligned}
$$

for large $n$.

Sequential Probability Assignment. Now, we are ready to present our probability assignment algorithm. We start with formula (7) on the mixture $M_{n}\left(x^{n}\right)$. Then we observe that $M_{n}\left(x_{n+1} \mid x^{n}\right)=M_{n}\left(x^{n+1}\right) / M_{n}\left(x^{n}\right)$. For example, if assume that $x_{n+1}$ symbol is $i \in \mathcal{A}$. Thus

$$
\begin{gathered}
M_{n}\left(x^{n+1}\right)=\frac{B\left(k_{1}+1 / 2, \cdots, k_{i}+3 / 2, \cdots, k_{m}+1 / 2\right)}{C(\mathcal{S}) \cdot B(\mathbf{1} / \mathbf{2})} \\
\cdot \operatorname{Dir}\left(\mathcal{S} ; k_{1}+1 / 2, \cdots, k_{i}+3 / 2, \cdots, k_{m}+1 / 2\right) .
\end{gathered}
$$

Using the functional equation of the gamma function, namely $\Gamma(x+1)=x \Gamma(x)$ allows us to write a simple sequential update algorithm that we present next. 
Corollary 1. Suppose that $m$ is fixed and that $\mathcal{S} \subseteq \Theta$ is a convex polytope. Let $N_{i}\left(x^{n}\right)$ be the number of symbol $i$ in $x^{n}$. Then

$$
\begin{gathered}
M_{n}\left(x_{n+1} \mid x^{n}\right)=\frac{N_{x_{n+1}}\left(x^{n}\right)+1 / 2}{n+m / 2} . \\
\frac{\operatorname{Dir}\left(\mathcal{S} ; N_{i}\left(x^{n}\right)+1 / 2+1\left(x_{n+1}=i\right), i=1 \cdots m\right)}{\operatorname{Dir}\left(\mathcal{S} ; N_{i}\left(x^{n}\right)+1 / 2, i=1 \cdots m\right)}
\end{gathered}
$$

which is the generalized add-1/2-KT estimator.

Observe that for the unconstrained case $\operatorname{Dir}\left(\Theta ; N_{i}\left(x^{n}\right)+1 / 2+1\left(x_{n+1}=i\right), i=1 \cdots m\right)=$ $\operatorname{Dir}\left(\Theta ; N_{i}\left(x^{n}\right)+1 / 2, i=1 \cdots m\right)=1$, and then our estimation algorithm reduces to the KT-estimator, that is,

$$
M_{n}\left(x_{n+1} \mid X^{n}\right)=\frac{N_{x_{n+1}}\left(x^{n}\right)+1 / 2}{n+m / 2} .
$$

We also observe that for the binary alphabet we recover [17] update, namely

$$
\begin{aligned}
M_{n}\left(x_{n+1} \mid x^{n}\right)= & \frac{N_{x_{n+1}}\left(x^{n}\right)+1 / 2}{n+1}++\left(2 x_{n+1}-1\right) \frac{a_{1}^{N_{1}\left(x^{n}\right)+1 / 2}\left(1-a_{1} N_{0}\left(x^{n}\right)+1 / 2\right.}{C(\mathcal{S})(n+1)} \\
& -\left(2 x_{n+1}-1\right) \frac{b_{1}^{N_{1}\left(x^{n}\right)+1 / 2}\left(1-b_{1} N_{0}\left(x^{n}\right)+1 / 2\right.}{C(\mathcal{S})(n+1)} .
\end{aligned}
$$

where $C(\mathcal{S})$ is defined in $(6)$. We should point out that the binary sequences sequential probability assignment as above was derived in [17] using a different technique that seems to be working only for binary sequences.

\section{Analysis and Proofs}

In this section we prove of our main results Theorems 1-3. Some technical details are delayed till the Appendix. We start with Theorem 2 since shall use some ideas and calculations of the proof of Theorem 2 in the proof of Theorem 1.

\subsection{Proof of Theorem 2}

We now prove Theorem 2 where we assume that $m$ may be large and $\mathcal{S}=\Theta$. By definition, we have $R_{n}^{*}(\Theta)=\log D_{n}$, where

$$
D_{n}=\sum_{\mathbf{k}}\left(\begin{array}{l}
n \\
\mathbf{k}
\end{array}\right) \prod_{i=1}^{m}\left(\frac{k_{i}}{n}\right)^{k_{i}}
$$

and the sum is taken over all non-negative integer vectors $\mathbf{k}=\left(k_{1}, \ldots, k_{m}\right)$ with $\sum_{i} k_{i}=n$. If we set

$$
S_{m}(n)=\sum_{\mathbf{k}}^{\prime}\left(\begin{array}{l}
n \\
\mathbf{k}
\end{array}\right) \prod_{i=1}^{m}\left(\frac{k_{i}}{n}\right)^{k_{i}}
$$


in which sum $\sum^{\prime}$ is taken over all $m$-dimensional integer vectors $\mathbf{k}=\left(k_{1}, \ldots, k_{m}\right)$ with $k_{j} \geq 1$ $(1 \leq j \leq m)$ and $k_{1}+\cdots+k_{m}=n$, then we can represent $D_{n}$ as

$$
D_{n}=(2 \pi)^{-\frac{m-1}{2}} \sqrt{n}\left(1+O\left(\frac{1}{n}\right)\right) \sum_{r=0}^{m-1}\left(\begin{array}{c}
m \\
r
\end{array}\right)(2 \pi)^{r / 2} S_{m-r}(n),
$$

that is, $S_{m-r}(n)$ takes care of those $\mathbf{k}$, where precisely $r$ components are zero.

By Stirling's formula we have $k^{k} e^{-k} \sqrt{2 \pi k} e^{1 /(12 k+1)}<k !<k^{k} e^{-k} \sqrt{2 \pi k} e^{1 /(12 k)}$ for all $k \geq 1$. Hence $S_{m}(n)$ satisfies

$$
\sum_{\mathbf{k}}^{\prime} \frac{1}{\sqrt{k_{1} \cdots k_{m}}} e^{-\sum_{i=1}^{m} \frac{1}{12 k_{i}+1}}<S_{m}(n)<\sum_{\mathbf{k}}^{\prime} \frac{1}{\sqrt{k_{1} \cdots k_{m}}} e^{-\sum_{i=1}^{m} \frac{1}{12 k_{i}}}
$$

By a standard but tedious analysis (see Section 4.2 of Appendix) we find the asymptotic relation

$$
S_{m}(n)=n^{\frac{m}{2}-1} B_{m}(\mathbf{1} / \mathbf{2})\left(1+O\left(\frac{m^{3 / 2}}{\sqrt{n}}\right)\right)
$$

as well as the upper bound

$$
S_{m}(n)=O\left(n^{\frac{m}{2}-1} B_{m}(\mathbf{1} / \mathbf{2})\right),
$$

where we recall that $B_{m}(\mathbf{1} / \mathbf{2})=B(\mathbf{1} / \mathbf{2})=\Gamma\left(\frac{1}{2}\right)^{m} / \Gamma\left(\frac{m}{2}\right)$.

Then (18) and (19) and the relation $B_{m-r}(\mathbf{1} / \mathbf{2})=O\left((m / \pi)^{r / 2} B_{m}(\mathbf{1} / \mathbf{2})\right)$ imply

$$
\begin{aligned}
D_{n} & =(2 \pi)^{-\frac{m-1}{2}} n^{\frac{m-1}{2}} B_{m}(\mathbf{1} / \mathbf{2})\left(1+O\left(\frac{m^{3 / 2}}{\sqrt{n}}\right)\right) \\
& +(2 \pi)^{-\frac{m-1}{2}} \sqrt{n} \sum_{r=1}^{m-1}\left(\begin{array}{c}
m \\
r
\end{array}\right) O\left((2 \pi)^{r / 2} B_{m-r}(\mathbf{1} / \mathbf{2}) n^{-r / 2}\right) \\
& =(2 \pi)^{-\frac{m-1}{2}} n^{\frac{m-1}{2}} B_{m}(\mathbf{1} / \mathbf{2})\left(1+O\left(\sqrt{\frac{m}{n}}\right)\right)^{m} \\
& +O\left((2 \pi)^{-\frac{m-1}{2}} n^{\frac{m-1}{2}} B_{m}(\mathbf{1} / \mathbf{2}) \frac{m^{3 / 2}}{\sqrt{n}}\right) \\
& =(2 \pi)^{-\frac{m-1}{2}} n^{\frac{m-1}{2}} B_{m}(\mathbf{1} / \mathbf{2}) \exp \left(O\left(\frac{m^{3 / 2}}{\sqrt{n}}\right)\right)
\end{aligned}
$$

Since

$$
\left.\log B_{m}(\mathbf{1} / \mathbf{2})=m \log \Gamma(1 / 2)-\log \Gamma(m / 2) m\right)
$$

we directly obtain the proposed representation (12) for $R_{n}^{*}(\Theta)=\log D_{n}$.

For the asymptotic average minimax $\bar{R}_{n}^{\text {asymp }}(\Theta)$ our starting point is

$$
\bar{R}_{n}^{\text {asymp }}(\Theta)=\frac{1}{B(\mathbf{1} / \mathbf{2})} \int_{\Theta} \sum_{\mathbf{k}}\left(\begin{array}{l}
n \\
\mathbf{k}
\end{array}\right) \boldsymbol{\theta}^{\mathbf{k}-\mathbf{1} / \mathbf{2}} \log \left(\frac{\boldsymbol{\theta}^{\mathbf{k}} B(\mathbf{1} / \mathbf{2})}{B(\mathbf{k}+\mathbf{1} / \mathbf{2})}\right)
$$


where we write $\boldsymbol{\theta}^{\mathbf{k}-\mathbf{1} / \mathbf{2}}:=\prod_{i} \theta_{i}^{k_{i}-1 / 2}$. We need to estimate different parts of the above sum. We first observe that

$$
\begin{aligned}
\sum_{\mathbf{k}}\left(\begin{array}{l}
n \\
\mathbf{k}
\end{array}\right) B(\mathbf{k}+\mathbf{1} / \mathbf{2}) & =\int_{\Theta} \sum_{\mathbf{k}}\left(\begin{array}{l}
n \\
\mathbf{k}
\end{array}\right) \boldsymbol{\theta}^{\mathbf{k}-\mathbf{1} / \mathbf{2}} d \boldsymbol{\theta} \\
& =\int_{\Theta} \boldsymbol{\theta}^{-\mathbf{1} / \mathbf{2}} d \boldsymbol{\theta}=B(\mathbf{1} / \mathbf{2})
\end{aligned}
$$

More importantly we notice that

$$
\begin{aligned}
\int_{\Theta} \sum_{\mathbf{k}}\left(\begin{array}{l}
n \\
\mathbf{k}
\end{array}\right) \boldsymbol{\theta}^{\mathbf{k}-\mathbf{1} / \mathbf{2}} \log \boldsymbol{\theta}^{\mathbf{k}} d \boldsymbol{\theta} & =\sum_{\mathbf{k}}\left(\begin{array}{l}
n \\
\mathbf{k}
\end{array}\right) \sum_{i=1}^{m} \int_{\Theta} \boldsymbol{\theta}^{\mathbf{k}-\mathbf{1} / \mathbf{2}} \log \theta_{i} d \boldsymbol{\theta} \\
& =\sum_{\mathbf{k}}\left(\begin{array}{l}
n \\
\mathbf{k}
\end{array}\right) \sum_{i=1}^{m} k_{i} \frac{\partial}{\partial k_{i}} B(\mathbf{k}+\mathbf{1} / \mathbf{2}) \int_{\Theta} \sum_{\mathbf{k}}\left(\begin{array}{l}
n \\
\mathbf{k}
\end{array}\right) \boldsymbol{\theta}^{\mathbf{k}-\mathbf{1} / \mathbf{2}} \log B(\mathbf{k}+\mathbf{1} / \mathbf{2}) \\
& =\sum_{\mathbf{k}}\left(\begin{array}{l}
n \\
\mathbf{k}
\end{array}\right) B(\mathbf{k}+\mathbf{1} / \mathbf{2}) \log B(\mathbf{k}+1 / 2) .
\end{aligned}
$$

Thus

$$
\bar{R}_{n}^{\text {asymp }}(\Theta)=\log B(\mathbf{1} / \mathbf{2})+\frac{1}{B(\mathbf{1} / \mathbf{2})} \sum_{\mathbf{k}}\left(\begin{array}{l}
n \\
\mathbf{k}
\end{array}\right) B(\mathbf{k}+\mathbf{1} / \mathbf{2}) \cdot\left(\sum_{i=1}^{m} k_{i} \frac{\partial}{\partial k_{i}} B(\mathbf{k}+\mathbf{1} / \mathbf{2})-\log B(\mathbf{k}+\mathbf{1} / \mathbf{2})\right) .
$$

To deal with such sums we use the relation between the beta function, the gamma function, and the psi function [19]. For example

$$
\frac{\partial}{\partial k_{i}} B(\mathbf{k}+\mathbf{1} / \mathbf{2})=\Psi\left(k_{i}+1 / 2\right)-\Psi(n+m / 2)
$$

where $\Psi(x)=\Gamma^{\prime}(x) / \Gamma(x)$. Asymptotically we have

$$
\begin{aligned}
\Psi(x+1 / 2) & =\log x+1 /(12 x)+O\left(1 / x^{3}\right), \\
\Psi(x+m / 2) & =\log x+(m-1) /(2 x)+O\left(1 / x^{2}\right) .
\end{aligned}
$$

Using this and Stirling's formula we find

$$
\begin{aligned}
\log \Gamma(x+1 / 2) & =x \log x-x+\log \sqrt{2 \pi}-\frac{1}{24 x}+O\left(1 / x^{2}\right), \\
\log \Gamma(x+m / 2) & =x \log x-x+\frac{m-1}{2} \log (x+m / 2) \\
& +\log \sqrt{2 \pi}+\left(\frac{1}{12}-\frac{m^{2}}{8}\right) \frac{1}{x}+O\left(m^{3} / x^{2}\right)
\end{aligned}
$$

leading to

$$
\begin{aligned}
\sum_{i=1}^{m} k_{i} \frac{\partial}{\partial k_{i}} B(\mathbf{k}+\mathbf{1} / \mathbf{2})-\log B(\mathbf{k}+\mathbf{1} / \mathbf{2}) & =\frac{m-1}{2}(\log (n+m / 2)-1-\log (2 \pi)) \\
& +O\left(m^{2} / n\right)+O\left(\sum_{i}\left(k_{i}+1\right)^{-1}\right) .
\end{aligned}
$$


Hence we obtain similarly to (32)

$$
\sum_{\mathbf{k}}\left(\begin{array}{l}
n \\
\mathbf{k}
\end{array}\right) \frac{B(\mathbf{k}+\mathbf{1} / \mathbf{2})}{k_{i}+1}=O\left(\frac{\sqrt{m} B(\mathbf{1} / \mathbf{2})}{\sqrt{n}}\right) .
$$

Summing up we arrive at

$$
\bar{R}_{n}^{\text {asmp }}(\Theta)=\frac{m-1}{2} \log (n / 2 \pi e)+\log \frac{\Gamma^{m}(1 / 2)}{\Gamma(m / 2)}+O\left(m^{3 / 2} / \sqrt{n}\right) .
$$

We now use Stirling's formula for $\Gamma(m / 2)$ and complete the proof.

\subsection{Proof of Theorem 1}

Recall that $R_{n}^{*}(\mathcal{S})=\log D_{n}$ where

$$
D_{n}=\sum_{\mathbf{k}}\left(\begin{array}{l}
n \\
\mathbf{k}
\end{array}\right) \sup _{\theta \in \mathcal{S}} \prod_{i=1}^{m} \theta_{i}^{k_{i}} .
$$

The problem is now that we have to distinguish between the case, where $\mathbf{k} / n \in \mathcal{S}$ and the case, where $\mathbf{k} / n \notin \mathcal{S}$. If $\mathbf{k} / n \in \mathcal{S}$ then we have

$$
\sup _{\theta \in \mathcal{S}} \prod_{i=1}^{m} \theta_{i}^{k_{i}}=\prod_{i=1}^{m}\left(\frac{k_{i}}{n}\right)^{k_{i}}
$$

as in the unconstrained case. If $\mathbf{k} / n \notin \mathcal{S}$ then we have

$$
\sup _{\theta \in \mathcal{S}} \prod_{i=1}^{m} \theta_{i}^{k_{i}}=\prod_{i=1}^{m} \theta_{i, \mathrm{opt}}^{k_{i}}
$$

where $\left(\theta_{i, \text { opt }}\right)$ is on the boundary of $\mathcal{S}$.

Let us first assume that $\mathbf{k} / n \in \mathcal{S}$. Then

$$
\begin{aligned}
D_{n}^{(\mathcal{S})} & :=\sum_{\mathbf{k} / n \in \mathcal{S}}\left(\begin{array}{l}
n \\
\mathbf{k}
\end{array}\right) \prod_{i=1}^{m}\left(\frac{k_{i}}{n}\right)^{k_{i}} \\
& =\left(\frac{n}{2 \pi}\right)^{\frac{1}{2}} C(\mathcal{S}) B(\mathbf{1} / \mathbf{2})(1+O(1 / \sqrt{n})) .
\end{aligned}
$$

The sum over $\mathbf{k}$ for which $\mathbf{k} / n \notin \mathcal{S}$ is more difficult to handle. But if $\mathcal{S}$ is a convex polytope we obtain after some (involved) algebra (see the Appendix)

$$
D_{n}-D_{n}^{(\mathcal{S})}=O\left(n^{\frac{m}{2}-1}\right)
$$

We just mention here the (trivial) case $m=2$ with $\mathcal{S}=\{(\theta, 1-\theta): \theta \in[a, b]\}$. Then

$$
\sup _{0 \leq \theta \leq 1} \theta^{k_{1}}(1-\theta)^{n-k_{1}}=a^{k_{1}}(1-a)^{n-k_{1}}
$$


and similar for $b n<k_{1} \leq n$. Furthermore,

$$
\begin{aligned}
D_{n}-D_{n}^{(\mathcal{S})} & =\sum_{0 \leq k_{1}<a n}\left(\begin{array}{c}
n \\
k_{1}
\end{array}\right) a^{k_{1}}(1-a)^{n-k_{1}} \\
& +\sum_{b n<k_{1} \leq n}\left(\begin{array}{c}
n \\
k_{1}
\end{array}\right) b^{k_{1}}(1-b)^{n-k_{1}} \\
& =1+O(1 / \sqrt{n})=O(1) .
\end{aligned}
$$

By using $B(\mathbf{1} / \mathbf{2})=\Gamma(1 / 2)^{m} / \Gamma(m / 2)$ and $\Gamma(1 / 2)=\sqrt{\pi}$ we directly obtain (10).

To prove the second statement of Theorem 1 , the starting point for the asymptotic average redundancy is (8), however, we rewrite it in terms of $\mathcal{S} \subseteq \Theta$ as follows

$$
\bar{R}_{n}^{\text {asympt }}(\mathcal{S})=\frac{1}{B_{\mathcal{S}}(\mathbf{1} / \mathbf{2})} \int_{\mathcal{S}} \sum_{\mathbf{k}}\left(\begin{array}{l}
n \\
\mathbf{k}
\end{array}\right) \boldsymbol{\theta}^{\mathbf{k}-\mathbf{1} / \mathbf{2}} \log \left(\frac{\boldsymbol{\theta}^{\mathbf{k}} B_{\mathcal{S}}(\mathbf{1} / \mathbf{2})}{B_{\mathcal{S}}(\mathbf{k}+\mathbf{1} / \mathbf{2})}\right)
$$

where we use the short hand notation

$$
B_{\mathcal{S}}(\boldsymbol{\alpha})=\int_{\mathcal{S}} \mathbf{x}^{\boldsymbol{\alpha}-1} d \mathbf{x}=\operatorname{Dir}(\mathcal{S} ; \boldsymbol{\alpha}) B(\boldsymbol{\alpha}) .
$$

As in the proof of Theorem 2 we obtain

$$
\bar{R}_{n}^{\text {asympt }}(\mathcal{S})=\log B_{\mathcal{S}}(\mathbf{1} / \mathbf{2})+\sum_{\mathbf{k}}\left(\begin{array}{l}
n \\
\mathbf{k}
\end{array}\right) \frac{B_{\mathcal{S}}(\mathbf{k}+\mathbf{1} / \mathbf{2})}{B_{\mathcal{S}}(\mathbf{1} / \mathbf{2})}\left(\sum_{i=1}^{m} k_{i} \frac{\partial}{\partial k_{i}} B_{\mathcal{S}}(\mathbf{k}+\mathbf{1} / \mathbf{2})-\log B_{\mathcal{S}}(\mathbf{k}+\mathbf{1} / \mathbf{2})\right) .
$$

Again we split the summation over $\mathbf{k}$ into several parts. If $\mathbf{k} / n \in \mathcal{S}^{-}$, where $\mathcal{S}^{-}$denotes all points in the interior of $\mathcal{S}$ with distance $\geq n^{-1 / 2+\varepsilon}$ to the boundary (for some $\varepsilon>0$ ), then the saddle point $\theta_{i}=k_{i} / n$ of the integrand $\overline{\boldsymbol{\theta}}^{\mathbf{k}}$ of the integral of $B_{\mathcal{S}}(\mathbf{k}+\mathbf{1} / \mathbf{2})$ or $\frac{\partial}{\partial k_{i}} B(\mathbf{k}+\mathbf{1} / \mathbf{2})$, respectively, is contained in $\mathcal{S}^{-}$. Consequently we find for any $L>0$

$$
\begin{gathered}
B_{\mathcal{S}}(\mathbf{k}+\mathbf{1} / \mathbf{2})=B(\mathbf{k}+\mathbf{1} / \mathbf{2})\left(1+O\left(n^{-L}\right)\right), \\
\frac{\partial}{\partial k_{i}} B_{\mathcal{S}}(\mathbf{k}+\mathbf{1} / \mathbf{2})=\frac{\partial}{\partial k_{i}} B(\mathbf{k}+\mathbf{1} / \mathbf{2})\left(1+O\left(n^{-L}\right)\right) .
\end{gathered}
$$

Hence

$$
\begin{aligned}
\sum_{\mathbf{k} / n \in \mathcal{S}^{-}}\left(\begin{array}{l}
n \\
\mathbf{k}
\end{array}\right) & B_{\mathcal{S}}(\mathbf{k}+\mathbf{1} / \mathbf{2})\left(\sum_{i=1}^{m} k_{i} \frac{\partial}{\partial k_{i}} B_{\mathcal{S}}(\mathbf{k}+\mathbf{1} / \mathbf{2})-\log B_{\mathcal{S}}(\mathbf{k}+\mathbf{1} / \mathbf{2})\right) \\
& =\sum_{\mathbf{k} / n \in \mathcal{S}^{-}}\left(\begin{array}{l}
n \\
\mathbf{k}
\end{array}\right) B(\mathbf{k}+\mathbf{1} / \mathbf{2})\left(\sum_{i=1}^{m} k_{i} \frac{\partial}{\partial k_{i}} B(\mathbf{k}+\mathbf{1} / \mathbf{2})-\log B(\mathbf{k}+\mathbf{1} / \mathbf{2})\right)+O\left(n^{-L}\right) \\
& =\sum_{\mathbf{k} / n \in \mathcal{S}^{-}}\left(\begin{array}{l}
n \\
\mathbf{k}
\end{array}\right) B(\mathbf{k}+\mathbf{1} / \mathbf{2}) \cdot\left(\frac{m-1}{2} \log \frac{n}{2 \pi e}+O\left(\sum_{i=1}^{m} 1 /\left(k_{i}+1\right)\right)\right)+O\left(n^{-L}\right) \\
& =\left(\frac{m-1}{2} \log \frac{n}{2 \pi e}+O(1 / \sqrt{n})\right) B_{\mathcal{S}}(\mathbf{1} / \mathbf{2}) .
\end{aligned}
$$


The other parts of the summation over $\mathbf{k}$ are more difficult to handle. We explain our approach for $m=2$ and $\mathcal{S}=\{(\theta, 1-\theta): \theta \in[a, b]\}$. Suppose that $\left|k_{1}-n b\right| \leq n^{1 / 2+\varepsilon}$, that is $\left(k_{1} / n, 1-k_{1} / n\right)$ is at distance $\leq n^{-1 / 2+\varepsilon}$ from the boundary of $\mathcal{S}$. Here we have

$$
B_{\mathcal{S}}\left(k_{1}+1 / 2, n-k_{1}+1 / 2\right)=\sqrt{\frac{2 \pi}{n}}\left(\frac{k_{1}}{n}\right)^{k_{1}}\left(\frac{n-k_{1}}{n}\right)^{n-k_{1}}\left(\Phi\left(\frac{n b-k_{1}}{\sqrt{n b(1-b)}}\right)+O(1 / \sqrt{n})\right),
$$

where $\Phi(u)$ denotes the normal distribution function. A similar representation holds for the derivatives $\frac{\partial}{\partial k_{i}} B_{\mathcal{S}}(\mathbf{k}+\mathbf{1} / \mathbf{2})$. After some algebra it follows that

$$
\sum_{\left|k_{1}-n b\right| \leq n^{1 / 2+\varepsilon}}\left(\begin{array}{l}
n \\
\mathbf{k}
\end{array}\right) B_{\mathcal{S}}(\mathbf{k}+\mathbf{1} / \mathbf{2}) \cdot\left(\sum_{i=1}^{m} k_{i} \frac{\partial}{\partial k_{i}} B_{\mathcal{S}}(\mathbf{k}+\mathbf{1} / \mathbf{2})-\log B_{\mathcal{S}}(\mathbf{k}+\mathbf{1} / \mathbf{2})\right)=O(1 / \sqrt{n}) .
$$

The summation for $n b+n^{1 / 2+\varepsilon}<k_{1} \leq n$ is much easier to handle, so we skip it.

For dimension $m>2$ one has to handle multivariate Gaussian approximations. This is just technical and more involved but there is no substantial problem. Summing up, in all cases the remainder is of order $O(1 / \sqrt{n})$. This completes the proof of Theorem 1 .

\subsection{Proof of Theorem 3}

The goal is to estimate the sum $D_{n}=D_{n}(\mathcal{M})$ :

$$
\begin{aligned}
D_{n} & =\sum_{\mathbf{k}}\left(\begin{array}{l}
n \\
\mathbf{k}
\end{array}\right) \max _{\theta_{1} \leq \theta_{2} \leq \cdots \leq \theta_{m}} \theta_{1}^{k_{1}} \theta_{2}^{k_{2}} \cdots \theta_{m}^{k_{m}} \\
& =\sum_{\mathbf{k} \in n \mathcal{M}}\left(\begin{array}{l}
n \\
\mathbf{k}
\end{array}\right)\left(\begin{array}{l}
n \\
\mathbf{k}
\end{array}\right) \prod_{i=1}^{m}\left(\frac{k_{i}}{n}\right)^{k_{1}}+\sum_{\mathbf{k} \notin n \mathcal{M}}\left(\begin{array}{l}
n \\
\mathbf{k}
\end{array}\right) \max _{\theta_{1} \leq \theta_{2} \leq \cdots \leq \theta_{m}} \theta_{1}^{k_{1}} \theta_{2}^{k_{2}} \cdots \theta_{m}^{k_{m}} \\
& =D_{n}^{(\mathcal{M})}+D_{n}^{(\Theta \backslash \mathcal{M})} .
\end{aligned}
$$

First let us consider the first part $D_{n}^{(\mathcal{M})}$ that we (again) partition into two parts. We set

$$
\mathcal{M}^{<}=\left\{\mathbf{k} \in n \mathcal{M}: k_{1}<k_{2}<\cdots k_{m}\right\}
$$

and then

$$
D_{n}^{(\mathcal{M})}=D_{n}^{\left(\mathcal{M}^{<}\right)}+D_{n}^{\left(\mathcal{M} \backslash \mathcal{M}^{<}\right)} .
$$

By using the fact that the functional $\prod_{i=1}^{m}\left(\frac{k_{i}}{n}\right)^{k_{1}}$ is symmetric it follows that the unconstrained sum (that we denote by $D_{n}(\Theta)$ ) is given by

$$
D_{n}(\Theta)=\sum_{\mathbf{k}}\left(\begin{array}{l}
n \\
\mathbf{k}
\end{array}\right) \prod_{i=1}^{m}\left(\frac{k_{i}}{n}\right)^{k_{1}}=m ! D_{n}^{\left(\mathcal{M}^{<}\right)}+\sum_{k_{i}=k_{j} \text { for some } i \neq j}\left(\begin{array}{l}
n \\
\mathbf{k}
\end{array}\right) \prod_{i=1}^{m}\left(\frac{k_{i}}{n}\right)^{k_{1}} .
$$

Now we have

$$
\sum_{k_{i}=k_{j} \text { for some } i \neq j}\left(\begin{array}{l}
n \\
\mathbf{k}
\end{array}\right) \prod_{i=1}^{m}\left(\frac{k_{i}}{n}\right)^{k_{i}} \leq\left(\begin{array}{c}
m \\
2
\end{array}\right) \sum_{k_{1}=k_{2}}\left(\begin{array}{l}
n \\
\mathbf{k}
\end{array}\right) \prod_{i=1}^{m}\left(\frac{k_{i}}{n}\right)^{k_{i}} .
$$


With the help of the approximation for $k_{i} \geq 1$ and $k_{1}=k_{2}$ :

$$
\left(\begin{array}{l}
n \\
\mathbf{k}
\end{array}\right) \prod_{i=1}^{m}\left(\frac{k_{i}}{n}\right)^{k_{1}} \approx(2 \pi)^{-\frac{m-1}{2}} \sqrt{\frac{n}{k_{1}^{2} k_{3} \cdots k_{m}}}
$$

we arrive at

$$
\sum_{\mathbf{k}}^{\prime} \frac{1}{\sqrt{k_{1}^{2} k_{3} \cdots k_{m}}}=\sum_{k=1}^{n / 2} \frac{1}{k} S_{m-2}^{(1)}(n-2 k)
$$

where $S_{n}^{(1)}(m)$ is defined as follows

$$
S_{m}^{(1)}(n)=\sum_{\mathbf{k}}^{\prime} \frac{1}{\sqrt{k_{1} \cdots k_{m}}}
$$

with the sum taken over all $m$-dimensional integer vectors $\mathbf{k}=\left(k_{1}, \ldots, k_{m}\right)$ with $k_{j} \geq 1$ $(1 \leq j \leq m)$ and $k_{1}+\cdots+k_{m}=n$. Using the upper bound (19) it follows that

$$
\sum_{k_{1}=k_{2}}\left(\begin{array}{l}
n \\
\mathbf{k}
\end{array}\right) \prod_{i=1}^{m}\left(\frac{k_{i}}{n}\right)^{k_{1}}=O\left((2 \pi)^{-\frac{m-1}{2}} \sqrt{m} n^{\frac{m-2}{2}} \log n B_{m}(\mathbf{1} / \mathbf{2}) \exp \left(O\left(\frac{m^{3 / 2}}{\sqrt{n}}\right)\right)\right) .
$$

Finally, by using (20) we get

$$
\begin{aligned}
D_{n}^{(\mathcal{M}<)} & =\frac{1}{m !}(2 \pi)^{-\frac{m-1}{2}} n^{\frac{m-1}{2}} B_{m}(\mathbf{1} / \mathbf{2}) \exp \left(O\left(\frac{m^{3 / 2}}{\sqrt{n}}\right)\right) \\
\times & \left(1+O\left(\frac{m^{5 / 2} \log n}{\sqrt{n}} \exp \left(O\left(\frac{m^{3 / 2}}{\sqrt{n}}\right)\right)\right)\right) .
\end{aligned}
$$

For the sum $D_{n}^{\left(\mathcal{M} \backslash \mathcal{M}^{<}\right)}$we use a simple estimate

$$
D_{n}^{\left(\mathcal{M} \backslash \mathcal{M}^{<}\right)} \leq(m-1) \sum_{k_{1}=k_{2}}\left(\begin{array}{l}
n \\
\mathbf{k}
\end{array}\right)\left(\frac{k_{i}}{n}\right)^{k_{i}}
$$

that can upper bounded by (27).

It remains to consider the sum $D_{n}^{(\Theta \backslash \mathcal{M})}$, where we sum over integer vectors $\left(k_{1}, \ldots, k_{m}\right)$ for which there exists $i$ with $k_{i}>k_{k+1}$. Due to the concavity property of the term $\prod_{i} \theta_{i}^{k_{i}}$ (that has optimum outside of $\mathcal{M})$ it follows that the optimum $\left(\theta_{1}^{\text {opt }}, \ldots, \theta_{m}^{\text {opt }}\right) \in \mathcal{M}$ of

$$
\prod_{i=1}^{m}\left(\theta_{1}^{\mathrm{opt}}\right)^{k_{i}}=\max _{\left(\theta_{1}, \ldots, \theta_{m}\right) \in \mathcal{M}} \prod_{i=1}^{m} \theta_{i}^{k_{i}}
$$

has to be on the boundary of $\mathcal{M}$, so we either have $\theta_{1}=0$ or $\theta_{j}=\theta_{j+1}$ for some $j=1, \ldots, m-1$. Hence we trivially have

$$
\begin{aligned}
\max _{\left(\theta_{1}, \ldots, \theta_{m}\right) \in \mathcal{M}} \prod_{i=1}^{m} \theta_{i}^{k_{i}} & \leq 0^{k_{1}} \max _{\theta_{2}+\cdots+\theta_{m-1}=1} \prod_{i=2}^{m} \theta_{i}^{k_{i}} \\
& +\sum_{j=1}^{m-1} \max _{\theta_{1}+\cdots+\theta_{j-1}+2 \theta_{j}+\theta_{j+2}+\cdots \theta_{m}=1} \prod_{i<j} \theta_{i}^{k_{i}} \cdot \theta_{j}^{k_{j}+k_{j+1}} \cdot \prod_{i>j+1} \theta_{i}^{k_{i}} .
\end{aligned}
$$


The first part is only non-zero if $k_{1}=0$. So it simplifies to the $m-1$-dimensional case. For the second part we note that

$$
\sum_{k_{j}+k_{j+1}=K}\left(\begin{array}{c}
n \\
k_{1} \cdots k_{m}
\end{array}\right)=2^{K}\left(\begin{array}{c}
n \\
k_{1} \cdots k_{j-1} K k_{j+2} \cdots k_{m}
\end{array}\right) .
$$

Thus, we are led to the optimize

$$
\max _{\theta_{1}+\cdots+\theta_{j-1}+2 \theta_{j}+\theta_{j+2}+\cdots \theta_{m}=1} \prod_{i<j}\left(\theta_{i}\right)^{k_{i}} \cdot\left(2 \theta_{j}\right)^{K} \cdot \prod_{i>j+1} \theta_{i}^{k_{i}}=\prod_{i<j}\left(\frac{k_{i}}{n}\right)^{k_{i}} \cdot\left(\frac{K}{n}\right)^{K} \cdot \prod_{i>j+1}\left(\frac{k_{i}}{n}\right)^{k_{i}} .
$$

So this case simplifies to the $m$-1-dimensional case, too. Summing up we have

$$
D_{n}^{(\Theta \backslash \mathcal{M})}=O\left((2 \pi)^{-\frac{m-1}{2}} m^{3 / 2} n^{\frac{m-2}{2}} B_{m}(\mathbf{1} / \mathbf{2}) \exp \left(O\left(\frac{m^{3 / 2}}{\sqrt{n}}\right)\right)\right) .
$$

Putting all together, we find

$$
\begin{aligned}
D_{n} & =\frac{1}{m !}(2 \pi)^{-\frac{m-1}{2}} n^{\frac{m-1}{2}} B_{m}(\mathbf{1} / \mathbf{2}) \exp \left(O\left(\frac{m^{3 / 2}}{\sqrt{n}}\right)\right) \\
& +O\left((2 \pi)^{-\frac{m-1}{2}} m^{3 / 2} n^{\frac{m-2}{2}} B_{m}(\mathbf{1} / \mathbf{2}) \exp \left(O\left(\frac{m^{3 / 2}}{\sqrt{n}}\right)\right)\right)
\end{aligned}
$$

This completes the proof of Theorem 3 .

\section{Appendix}

\subsection{Proof of (18) and 19)}

We suppose that $m \geq 2$ and $n \geq 1$ are integers. We first estimate $\left|S_{m}(n)-S_{m}^{(1)}(n)\right|$ where $S_{m}(n)$ and $S_{m}^{(1)}(n)$ are defined in (17) and (26), respectively. Since $1-e^{-x} \leq x$ we have

$$
\left|S_{m}(n)-S_{m}^{(1)}(n)\right| \leq \sum_{\mathbf{k}}^{\prime} \frac{1}{\sqrt{k_{1} \cdots k_{m}}} \sum_{i=1}^{m} \frac{1}{12 k_{i}}=\frac{m}{12} \sum_{\mathbf{k}}^{\prime} \frac{1}{\sqrt{k_{1} \cdots k_{m}}} \frac{1}{k_{1}}
$$

We note that we have the recurrence

$$
S_{m}^{(1)}(n)=\sum_{k=1}^{n-1} \frac{1}{\sqrt{k}} S_{m-1}^{(1)}(n-k) .
$$

and that

$$
B(\mathbf{1} / \mathbf{2})=B_{m}(\mathbf{1} / \mathbf{2})=\frac{\Gamma(1 / 2)^{m}}{\Gamma(m / 2)}=\frac{\pi^{m / 2}}{\Gamma(m / 2)}
$$

as well as

$$
B_{m-1}(\mathbf{1} / \mathbf{2}) B(1 / 2,(m-1) / 2)=B_{m}(\mathbf{1} / \mathbf{2}) .
$$

Furthermore, for $m \geq 3$ the function $x \mapsto x^{-\frac{1}{2}}(n-x)^{\frac{m-3}{2}}$ is decreasing that leads to the upper bound

$$
\sum_{k=1}^{n-1} \frac{1}{\sqrt{k}}(n-k)^{\frac{m-3}{2}} \leq \int_{0}^{n} \frac{1}{\sqrt{x}}(n-x)^{\frac{m-3}{2}} d x=n^{\frac{m-2}{2}} B(1 / 2,(m-1) / 2) .
$$


It is now easy to obtain (19) by induction. Clearly we have

$$
\sum_{k=1}^{n-1} \frac{1}{\sqrt{k(n-k)}} \leq C_{1}
$$

with a proper constant $C_{1}>0$. Thus, (19) holds for $m=2$. Furthermore, by using (30) and (31) we get inductively (for $m \geq 3$ )

$$
\begin{aligned}
S_{m}^{(1)}(n) & \leq \sum_{k=1}^{n-1} \frac{1}{\sqrt{k}} C_{1}(n-k)^{\frac{m-3}{2}} B_{m-1}(\mathbf{1} / \mathbf{2}) \\
& \leq C_{1} n^{\frac{m-2}{2}} B(1 / 2,(m-1) / 2) B_{m-1}(\mathbf{1} / \mathbf{2}) \\
& =C_{1} n^{\frac{m-2}{2}} B_{m}(\mathbf{1} / \mathbf{2}) .
\end{aligned}
$$

This upper bound also shows that (18) is true if $m \geq c n^{1 / 3}$ (for any positive constant $c>0$. Thus, it will remain to consider the case $m<c n^{1 / 3}$.

Another application of the upper bound (19) leading to an upper bound of

$$
\begin{aligned}
\sum_{\mathbf{k}} \frac{1}{\sqrt{k_{1} \cdots k_{m}}} \frac{1}{k_{1}} & =\sum_{k=1}^{n-1} \frac{1}{k \sqrt{k}} S_{m-1}^{(1)}(n-k) \\
& =O\left(B_{m-1}(\mathbf{1} / \mathbf{2}) \sum_{k=1}^{n-1} \frac{1}{k \sqrt{k}}(n-k)^{\frac{m-3}{2}}\right) .
\end{aligned}
$$

Since $B_{m-1}(\mathbf{1} / \mathbf{2})=O\left(\sqrt{m} B_{m}(\mathbf{1} / \mathbf{2})\right)$ and

$$
\sum_{k=1}^{n-1} \frac{1}{k \sqrt{k}}(n-k)^{\frac{m-3}{2}}=O\left(n^{\frac{m-3}{2}}\right)
$$

we directly obtain

$$
\sum_{\mathbf{k}} \frac{1}{\sqrt{k_{1} \cdots k_{m}}} \frac{1}{k_{1}}=O\left(B(\mathbf{1} / \mathbf{2}) m^{\frac{1}{2}} n^{\frac{m}{2}-\frac{3}{2}}\right) .
$$

Together with (29) we, thus find

$$
\left|S_{m}(n)-S_{m}^{(1)}(n)\right|=O\left(B(\mathbf{1} / \mathbf{2}) m^{\frac{3}{2}} n^{\frac{m}{2}-\frac{3}{2}}\right) .
$$

In order to complete the proof of (18) it remains to show that

$$
S_{m}^{(1)}(n)=n^{\frac{m}{2}-1} B_{m}(\mathbf{1} / \mathbf{2})\left(1+O\left(\frac{m^{3 / 2}}{\sqrt{n}}\right)\right)
$$

holds for $m<c n^{1 / 3}$.

For this purpose we use (again) induction and the simplest form of the Euler-MacLaurin formula [19], namely

$$
\sum_{n=a}^{b} f(n)=\int_{a}^{b} f(x) d x+\frac{f(a)+f(b)}{2}+\int_{a}^{b}\left(x-\lfloor x\rfloor-\frac{1}{2}\right) f^{\prime}(x) d x,
$$


where $f(x)$ is continuously differentiable and $a<b$ are integers. For example, we obtain the asymptotic relation

$$
\begin{aligned}
S_{2}^{(1)}(n) & =\sum_{k=1}^{n-1} \frac{1}{\sqrt{k(n-k)}} \\
& =\int_{1}^{n-1} \frac{d x}{\sqrt{x(n-x)}}+O\left(n^{-1 / 2}\right)+O\left(\int_{1}^{n-1} \frac{d x}{\sqrt{x^{3}(n-x)}}\right) \\
& =B(1 / 2,1 / 2)+O\left(n^{-1 / 2}\right)
\end{aligned}
$$

that is in accordance with (33).

Furthermore we find for $m \geq 3$

$$
\begin{aligned}
\sum_{k=1}^{n-1} \frac{1}{\sqrt{k}}(n-k)^{\frac{m-3}{2}} & =\int_{1}^{n-1} \frac{1}{\sqrt{x}}(n-x)^{\frac{m-3}{2}} d x+O\left(n^{\frac{m-3}{2}}\right) \\
& +O\left(\int_{1}^{n-1} x^{-3 / 2}(n-x)^{\frac{m-3}{2}} d x\right)+O\left(m \int_{1}^{n-1} x^{-1 / 2}(n-x)^{\frac{m-5}{2}} d x\right) \\
& =n^{\frac{m-2}{2}} B(1 / 2,(m-1) / 2)+O\left(n^{\frac{m-3}{2}}\right)+O\left(\sqrt{m} n^{\frac{m-4}{2}}\right) \\
& =n^{\frac{m-2}{2}} B(1 / 2,(m-1) / 2)\left(1+O\left(\sqrt{\frac{m}{n}}\right)\right) .
\end{aligned}
$$

With the help of these expansions we will prove (33) by induction on $m$. For notational convenience we write $O_{C}(X)$ for a term that is absolutely bounded by $\leq C|X|$, that is, we specify the implicit constant.

We already mentioned that (33) holds for $m=2$, see (34). Actually we can prove (33) for every fixed $m \geq 2$ (we just have to apply the inductive method a finite number of times). Hence, we can assume that (33) holds for $m \leq m_{0}$, where $m_{0} \geq 2$ is a fixed but arbitrary integer. Now suppose that $m>m_{0}$ and that (33) holds for $m-1$ :

$$
S_{m-1}^{(1)}(n)=n^{\frac{m-3}{2}} B_{m-1}(\mathbf{1} / \mathbf{2})\left(1+O_{C}\left(\frac{(m-1)^{3 / 2}}{\sqrt{n}}\right)\right) .
$$

By (30) we, thus, obtain

$$
\begin{aligned}
S_{m}^{(1)}(n) & =\sum_{k=1}^{n-1} \frac{1}{\sqrt{k}}(n-k)^{\frac{m-3}{2}} B_{m-1}(\mathbf{1} / \mathbf{2})\left(1+O_{C}\left(\frac{(m-1)^{3 / 2}}{\sqrt{n-k}}\right)\right) \\
& =n^{\frac{m-2}{2}} B(1 / 2,(m-1) / 2) B_{m-1}(\mathbf{1} / \mathbf{2})\left(1+O\left(\sqrt{\frac{m}{n}}\right)\right) \\
& +O_{C}\left((m-1)^{3 / 2} n^{\frac{m-3}{2}} B(1 / 2,(m-2) / 2) B_{m-1}(\mathbf{1} / \mathbf{2})\left(1+O\left(\sqrt{\frac{m}{n}}\right)\right)\right) .
\end{aligned}
$$

Now note that $(m-1)^{3 / 2} \leq m^{3 / 2}\left(1-c_{1} / m\right)$, where the constant $c_{1}>0$ is certainly $\leq \frac{3}{2}$. However, if $m$ is sufficiently large (say $m \geq m_{0}$ ) then we can assume that $c_{1}>1$. Furthermore we have

$$
B(1 / 2,(m-2) / 2) B_{m-1}(\mathbf{1} / \mathbf{2}) \leq B_{m}(\mathbf{1} / \mathbf{2})\left(1+c_{2} / m\right)
$$


where $c_{2}>\frac{1}{2}$. However, if $m$ is sufficiently large then we can assume that $c_{2}<\frac{2}{3}$. Consequently we have

$S_{m}^{(1)}(n)=n^{\frac{m-2}{2}} B_{m}(\mathbf{1} / \mathbf{2})\left(1+O\left(\sqrt{\frac{m}{n}}\right)+O_{C}\left(\frac{m^{3 / 2}}{\sqrt{n}}\left(1-\frac{c_{1}}{m}\right)\left(1+\frac{c_{2}}{m}\right)\left(1+O\left(\sqrt{\frac{m}{n}}\right)\right)\right)\right.$.

Recall that we only have to consider the case, where $m<c n^{3 / 2}$, where $c>0$ can be arbitrarily chosen. Since $c_{1}>1$ and $c_{2}<\frac{2}{3}$ we can choose $c>0$ properly such that

$$
\left(1-\frac{c_{1}}{m}\right)\left(1+\frac{c_{2}}{m}\right)\left(1+O\left(\sqrt{\frac{m}{n}}\right)\right) \leq 1-\frac{1}{4 m}
$$

for $m_{0} \leq m<c n^{3 / 2}$. Finally, by adjusting $C$ we also assure that

$$
O_{C}\left(\frac{m^{3 / 2}}{\sqrt{n}}\left(1-\frac{1}{4 m}\right)\right)+O\left(\sqrt{\frac{m}{n}}\right)=O_{C}\left(\frac{m^{3 / 2}}{\sqrt{n}}\right)
$$

for $m<c n^{3 / 2}$. Hence, (33) follows for all $m<c n^{3 / 2}$ and we are done.

\subsection{Proof of (23)}

As mentioned in Section 3.2 the analysis of the proof of (23) is very involved. We recall that a convex polytope is the intersection of finitely many half spaces. Thus, in order to cover $\Theta \backslash S$ we just have to consider the union of finitely many half spaces. Furthermore, by concavity of $\prod_{i} \theta_{i}^{k_{i}}$ it follows that the optimal choice of $\boldsymbol{\theta}=\left(\theta_{1}, \ldots, \theta_{m}\right)$ has to be on the boundary of $\mathcal{S}$. Therefore it is sufficient to consider just one half space $H$ and take the optimum $\boldsymbol{\theta}$ on the boundary $\partial H$.

Without loss of generality we can assume that the boundary $\partial H$ is given by the equation

$$
a_{1} \theta_{1}+a_{2} \theta_{2}+\cdots+a_{m-1} \theta_{m-1}=1
$$

where $a_{m-1} \neq 0$. (If all coefficients are non-zero we can use the equation $\theta_{1}+\cdots+\theta_{m}=1$ to eliminate one variable.) Of course we only consider half spaces with the property that $H \cap \Theta$ is a $m-2$-dimensional polytope of positive $m-2$-dimensional volume. For simplicity we also assume that $a_{j} \neq 1,1 \leq j \leq a_{m-1}$. The other cases can be treated similarly but the calculations are more involved.

We then analyze the following sum:

$$
S=\sum_{\mathbf{k} \in n(H \cap \Theta)}\left(\begin{array}{l}
n \\
\mathbf{k}
\end{array}\right) \max _{\boldsymbol{\theta} \in \partial H \cap \Theta} \prod_{i=1}^{m} \theta_{i}^{k_{i}}
$$

In order to simplify our considerations we replace the sum by an integral, that is, we consider $k_{i}$ as continuous variables. Since we are in a fixed dimension and we have several monotonicity properties it is easy to verify that this approximation is justified, even more if we are just interested in upper bounds.

Clearly if $\mathbf{k} \in n(\partial H \cap \Theta)$ then

$$
\max _{\boldsymbol{\theta} \in \partial H \cap \Theta} \prod_{i=1}^{m} \theta_{i}^{k_{i}}=\prod_{i=1}^{m}\left(\frac{k_{i}}{n}\right)^{k_{i}}
$$


Let's fix such a situation for some $\mathbf{k}_{0}=\left(k_{1,0}, \ldots, k_{m, 0}\right) \in n(\partial H \cap \Theta)$, that is, we have

$$
\sum_{i=1}^{m} k_{i, 0}=n \quad \text { and } \quad \sum_{i=1}^{m-1} a_{i} k_{i, 0}=n
$$

Furthermore we set $\theta_{i, 0}=k_{i, 0} / n$.

Suppose now that we are not on the boundary of $\partial H \cap \Theta$. For this case will we characterize those $\mathbf{k} \in n(H \cap \Theta)$ for which

$$
\max _{\boldsymbol{\theta} \in \partial H \cap \Theta} \prod_{i=1}^{m} \theta_{i}^{k_{i}}=\prod_{i=1}^{m} \theta_{i, 0}^{k_{i}} .
$$

(The boundary case has to be handled in a slightly different way but the contribution will be negligible.) For this purpose we parametrize $\boldsymbol{\theta} \in \partial H \cap \Theta$ by $\theta_{1}, \ldots, \theta_{m-2}$ so that $\theta_{m-1}$ and $\theta_{m}$ are given by

$$
\begin{aligned}
\theta_{m-1} & =\frac{1}{a_{m-1}}-\sum_{i=1}^{m-2} \frac{a_{i}}{a_{m-1}} \theta_{i}, \\
\theta_{m} & =\frac{a_{m-1}-1}{a_{m-1}}+\sum_{i=1}^{m-2} \frac{a_{i}-a_{m-1}}{a_{m-1}} \theta_{i} .
\end{aligned}
$$

Since our optimum is assumed to be not on the boundary of $\partial H \cap \Theta$ and by the concavity property of the mapping $\boldsymbol{\theta} \mapsto \sum_{i} k_{i} \log \theta_{i}$ it follows that $\mathbf{k}$ has to satisfy (besides (35)) the system of equations

$$
\frac{\partial}{\partial \theta_{i}} \sum_{i=1}^{m} k_{i} \log \theta_{i, 0}=\frac{k_{i}}{\theta_{i, 0}}-\frac{k_{m-1}}{\theta_{m-1,0}} \frac{a_{i}}{a_{m-1}}+\frac{k_{m}}{\theta_{m, 0}} \frac{a_{i}-a_{m-1}}{a_{m-1}}=0, \quad 1 \leq i \leq m-2 .
$$

Clearly $k_{i}=k_{i, 0}$ satisfy this system of equations.

We set $\ell_{i}=k_{i}-k_{i, 0}, 1 \leq i \leq m$. Then we certainly have $\sum_{i} \ell_{i}=0$ and

$$
\frac{\ell_{i}}{\theta_{i, 0}}-\frac{\ell_{m-1}}{\theta_{m-1,0}} \frac{a_{i}}{a_{m-1}}+\frac{\ell_{m}}{\theta_{m, 0}} \frac{a_{i}-a_{m-1}}{a_{m-1}}=0, \quad 1 \leq i \leq m-2,
$$

that is, we have $m-1$ homogeneous equations for $m$ variables. It is easy to check that the one dimensional solution can be parametrized by $\ell_{m}$ :

$$
\ell_{i}=\ell_{m} \frac{\theta_{i, 0}}{\theta_{m, 0}}\left(1-a_{i}\right), \quad 1 \leq i \leq m-1 .
$$

By the way, if we set $a_{m}=0$ then this is also true for $i=m$. Summing up, we have (36) if and only if $k_{i}=k_{i, 0}+\ell_{i}, 1 \leq i \leq m$, where $\ell_{i}$ are given by (37).

Since we have

$$
\begin{aligned}
\left(\begin{array}{c}
n \\
\mathbf{k}
\end{array}\right) \prod_{i=1}^{m} \theta_{i, 0}^{k_{i}} & =\left(\begin{array}{c}
n \\
\mathbf{k}_{0}
\end{array}\right) \prod_{i=1}^{m} \theta_{i, 0}^{k_{i, 0}} \prod_{i=1}^{m} \prod_{j=1}^{\ell_{i}} \frac{k_{i, 0}}{k_{i, 0}+j} \\
& \sim(2 \pi)^{-\frac{m-1}{2}} \sqrt{\frac{n}{k_{1,0} \cdots k_{m, 0}}} \exp \left(-\sum_{i=1}^{m} \frac{\ell_{1}^{2}}{2 k_{i, 0}}\right)
\end{aligned}
$$


for $k_{i}=k_{i, 0}+\ell_{i}, 1 \leq i \leq m$, where $\sum_{i} \ell_{i}=0$, and

$$
\begin{aligned}
\sum_{i=1}^{m} \frac{\ell_{1}^{2}}{2 k_{i, 0}} & =\frac{\ell_{m}^{2} n}{2 k_{m, 0}^{2}} \sum_{i=1}^{m}\left(1-a_{i}\right)^{2} \theta_{i, 0} \\
& \geq \frac{\ell_{m}^{2} n}{2 k_{m, 0}^{2}} \min _{i}\left(1-a_{i}\right)^{2}
\end{aligned}
$$

we can integrate over $\ell_{m}$ and obtain in a first step an upper bound for

$$
S=O\left((2 \pi)^{-\frac{m-1}{2}} \int_{K} \sqrt{\frac{k_{m, 0}}{k_{1,0} \cdots k_{m-1,0}}} d \mathbf{k}_{0}\right) .
$$

Here $K$ denotes the set of all $\left(k_{1,0}, \ldots, k_{m-2,0}\right)$ such that $\mathbf{k}_{0}=\left(k_{1,0}, \ldots, k_{m, 0}\right) \in n(\partial H \cap \Theta)$, where

$$
\begin{aligned}
k_{m-1,0} & =\frac{n}{a_{m-1}}-\sum_{i=1}^{m-2} \frac{a_{i}}{a_{m-1}} k_{i, 0}, \\
k_{m, 0} & =\frac{a_{m-1}-1}{a_{m-1}} n+\sum_{i=1}^{m-2} \frac{a_{i}-a_{m-1}}{a_{m-1}} k_{i, 0} .
\end{aligned}
$$

This directly leads to the upper bound

$$
S=O\left(n^{\frac{m-2}{2}}\right)
$$

as proposed in (23).

In order to make the arguments completely rigorous we have to consider also hyperplanes, where some of the coefficients $a_{i}$ equal 1 and to argue that we can estimate sums by integrals. However, this is just a technical difficulty and not a substantial problem.

\section{References}

[1] J. Bernardo, Reference Posterior Distributions for Bayesian Inference, J. Roy. Stat. Soc. B., 41, 113-147, 1979.

[2] B. S. Clarke and A. R. Barron, Asymptotic of Bayes Information's Theoretic Asymptotics of Bayes Methods, IEEE Trans. Inform. Theory, 36, 453-471, 1990.

[3] T. Cover and J.A. Thomas, Elements of Information Theory, John Wiley \& Sons, New York 1991.

[4] L. Davisson, Universal Noiseless Coding, IEEE Trans. Inform. Theory, 19, 783-795, 1973.

[5] M. Drmota and W. Szpankowski, Precise Minimax Redundancy and Regrets, IEEE Trans. Information Theory, 50, 2686-2707, 2004.

[6] P. Flajolet, Singularity analysis and asymptotics of Bernoulli sums, Theoretical Computer Science, 215, 371-381, 1999. 
[7] R. Gallager, Information Theory and Reliable Communication, John Wiley \& Sons, New York, 1968.

[8] P. Jacquet and W. Szpankowski, Entropy Computations via Analytic Depoissonization, IEEE Information Theory, 45, 1072-1081, 1999. Entropy Computations via Analytic Depoissonization, IEEE Information Theory, 45, 1072-1081, 1999.

[9] R. Krichevsky and V. Trofimov, The Performance of Universal Coding, IEEE Trans. Information Theory, 27, 199-207, 1983.

[10] A. Orlitsky and N. P. Santhanam, Speaking of Infinity, IEEE Transactions on Information Theory, 50, 2215-2230, 2004

[11] A. Orlitsky, P. Santhanam, and J. Zhang Universal Compression of Memoryless Sources over Unknown Alphabets, IEEE Trans. Information Theory, 50, 1469-1481, 2004.

[12] J. Rissanen, Fisher Information and Stochastic Complexity, IEEE Trans. Information Theory, 42, 40-47, 1996.

[13] Y. Shtarkov, Universal Sequential Coding of Single Messages, Problems of Information Transmission, 23, 175-186, 1987. Problems of Information Transmission, 23, 175-186, 1987.

[14] G. Shamir, Universal Lossless Compression with Unknown Alphabets - The Average Case IEEE Transactions on Information, 52, 4915-4944, 2006.

[15] G. Shamir, On the MDL Principle for i.i.d. Sources With Large Alphabets, IEEE Transactions on Information, 52, 1939-1955, 2006.

[16] G. Shamir, Universal Source Coding for Monotonic and Fast Decaying Monotonic Distributions, IEEE Transactions on Information Theory, 59, 7194-7211, 2013.

[17] G. Shamir, T. Tjalkens and F. Willems, Low-Complexity Sequential Probability Estimation and Universal Compression for Binary Sequences with Constrained Distributions, ISIT, 2008. with Constrained Distributions, ISIT, 2008.

[18] W. Szpankowski, On Asymptotics of Certain Recurrences Arising in Universal Coding, Problems of Information Transmission, 34, 55-61, 1998.

[19] W. Szpankowski, Average Case Analysis of Algorithms on Sequences, Wiley, New York, 2001.

[20] W. Szpankowski and M. Weinberger, Minimax Pointwise Redundancy for Memoryless Models over Large Alphabets, IEEE Trans. Information Theory, 58, 4094-4104, 2012.

[21] Q. Xie, A. Barron, Minimax Redundancy for the Class of Memoryless Sources, IEEE Trans. Information Theory, 43, 647-657, 1997.

[22] Q. Xie, A. Barron, Asymptotic Minimax Regret for Data Compression, Gambling, and Prediction, IEEE Trans. Information Theory, 46, 431-445, 2000. 